\title{
A Short-chain Dehydrogenase/reductase Gene is Required for Infection-related Development and Pathogenicity in Magnaporthe oryzae
}

\author{
Minjung Kwon ${ }^{\dagger}$ Kyoung Su Kim ${ }^{\dagger}$ and Yong-Hwan Lee* \\ Department of Agricultural Biotechnology, Center for Fungal Genetic Resources, and Center for Fungal Pathogenesis, Seoul \\ National University, Seoul 151-921, Korea \\ (Received on October 28, 2009; Accepted on November 22, 2009)
}

The phytopathogenic fungus Magnaporthe oryzae is a major limiting factor in rice production. To understand the genetic basis of $M$. oryzae pathogenic development, we previously analyzed a library of T-DNA insertional mutants of $M$. oryzae, and identified ATMT0879A1 as one of the pathogenicity-defective mutants. Molecular analyses and database searches revealed that a single $\mathrm{T}$ DNA insertion in ATMT0879A1 resulted in functional interference with an annotated gene, MGG00056, which encodes a short-chain dehydrogenase/reductase (SDR). The mutant and annotated gene were designated as MoSDR1 $1^{\text {T-DNA }}$ and MoSDR1, respectively. Like other SDR family members, MoSDR1 possesses both a cofactor-binding motif and a catalytic site. The expression pattern of MoSDR1 suggests that the gene is associated with pathogenicity and plays an important role in $M$. oryzae development. To understand the roles of MoSDRI, the deletion mutant $\Delta M o s d r 1$ for the gene was obtained via homology-dependent gene replacement. As expected, $\Delta M o s d r 1$ was nonpathogenic; moreover, the mutant displayed pleiotropic defects in conidiation, conidial germination, appressorium formation, penetration, and growth inside host tissues. These results suggest that MoSDRI functions as a key metabolic enzyme in the regulation of development and pathogenicity in $M$. oryzae.

Keywords : appressorium, conidiation, rice blast, short-chain dehydrogenase/reductase (SDR)

Magnaporthe oryzae is an ascomyceteous phytopathogen that causes rice blast, one of the most destructive diseases found in all rice-growing countries (Ou, 1985). The disease continues to be a major constraint in rice production, and it is exacerbated by such factors as global population growth. Development of the disease requires appropriate fungal development and pathogenicity, which are controlled by complicated cellular processes. Disease occurs when the

\footnotetext{
*Corresponding author.

Phone) +82-2-880-4674, FAX) +82-2-873-2317

E-mail) yonglee@snu.ac.kr

"equally contributed.
}

conidium (asexual spore) attaches to suitable host tissues. After conidial attachment, a germ tube emerges at the apex that differentiates into a specialized infectious structure, called an appressorium, upon its perception of host factors. Enormous internal turgor pressure $(>8 \mathrm{MPa})$ is generated inside the appressorium, which enables the fungus to penetrate host cells through mechanical disruption of the host's epidermal barriers (Howard et al., 1991). The fungus then develops invasive hyphae in the host cell membrane, colonizes whole cells, and escapes via conidiation.

M. oryzae has emerged as an important model organism for studying the molecular basis of fungal development and pathogenicity due to its genetic amenability, genomic sequence data, and the severe economic impact of rice blast (Dean et al., 2005; Valent, 1990). Several studies have shown that conserved core signaling pathways are associated with the transduction of signals into cellular responses for appressorium-mediated disease development. These include cyclic adenosine monophosphate (cAMP) (Choi and Dean, 1997; Mitchell and Dean, 1995), mitogen-activated protein (MAP) kinase (Jeon et al., 2008; Xu and Hamer, 1996; Xu et al., 1997), and $\mathrm{Ca}^{2+}$-dependent signaling pathways (Choi et al., 2009a; Choi et al., 2009b). Whereas the effects of conserved signaling pathways on $M$. oryzae development and pathogenicity are well documented, little is known of the other determinants and pathways that direct infectionrelated development and pathogenicity. Although successful disease development by $M$. oryzae involves intercellular signaling as well as metabolic and regulatory networks that are highly integrated, most studies have focused on signaling pathways without considering the interplay that exists between them.

Members of the short-chain dehydrogenase/reductase (SDR) family are present in all forms of life, from simple organisms to higher eukaryotes (Jörnvall et al., 1999), indicating their versatility and fundamental importance in metabolic processes (Persson et al., 2009). Most dehydrogenases (about 25\%) belong to the SDR family (Kallberg and Persson, 2006). As the first characterized member of the SDR family was Drosophila alcohol dehydrogenase, this family used to be called "insect-type" or "short-chain" 
alcohol dehydrogenase (Villarroya et al., 1989). SDRs are also known as $\mathrm{NAD}(\mathrm{P})(\mathrm{H})$-dependent oxidoreductases, which are distinct from the medium-chain dehydrogenase/ reductase (MDR) and aldo-keto reductase (AKR) superfamilies (Jörnvall et al., 1995). There are two conserved motifs in the SDR family. The first motif at the N-terminus defines the cofactor-binding site (TGxxxGxG). This region consists of alternating $\beta$-sheets and $\alpha$-helices $(\beta-\alpha-\beta)$, homologous to Rossmann-fold elements with a typical $\alpha / \beta$ folding pattern (Oppermann et al., 2001; Rossmann and Argos, 1978). The second region houses specific residues for the catalytic site (YxxxK), which is not present in the MDR or AKR superfamily (Jörnvall et al., 1995).

SDRs play diverse and important roles in cellular differentiation and signaling. SDRs are involved in retinoid and steroid hormone biosynthesis in mammals (Oppermann et al., 2001) and sex determination in maize by controlling cell death (DeLong et al., 1993). Other SDRs have been found to convert xanthoxin to abscisic acid (ABA)-aldehyde in ABA biosynthesis (Cheng et al., 2002), which is critical for plant growth, development, and stress responses. Members of the SDR family such as trihydroxynaphthalene reductase $(3 \mathrm{HNR})$ are key enzymes in fungal melanin biosynthesis (Thompson et al., 1997), which is required for pathogenicity in M. oryzae. Also, an SDR has been shown to be involved in the biosynthesis of fumonisin toxin in Gibberella moniliformis (Butchko et al., 2003).

To better understand M. oryzae development and pathogenicity, we previously generated a genome-wide mutant library via Agrobacterium tumefaciens-mediated transformation (ATMT) and screened mutants to find genes required for the disease development (Jeon et al., 2007). In this study, we characterized one of pathogenicity defective TDNA insertional mutants, ATMT0879A1, that is renamed here as MoSDR $1^{\mathrm{T}-\mathrm{DNA}}$. Deletion mutants of MoSDR1 were generated through homology-dependent gene replacement to confirm the involvement of MoSDRl in pathogenicity. Analyses of the $\triangle M o s d r l$ mutants revealed that MoSDRl is essential for conidiation, conidial germination, and appressorium development.

\section{Materials and Methods}

Fungal strains and culture conditions. $M$. oryzae strain KJ201, which was obtained from the Center for Fungal Genetic Resources (CFGR; http://cfgr.snu.ac.kr), was used as the wild-type strain. The wild-type strain and transformants generated in this study were cultured on either oatmeal agar medium [OMA; 5\% oatmeal (w/v) and 1.5\% agar] or V8 juice agar medium (4\% V8 juice and 1.5\% agar) at $25^{\circ} \mathrm{C}$ under constant fluorescent light to promote conidiation (Lee and Lee, 1998). Genomic DNA and total
RNA were extracted from four-day-old mycelia cultured in complete liquid medium $(0.6 \%$ yeast extract, $0.6 \%$ tryptone, and $1 \%$ sucrose) as described previously (Chi et al., 2009a).

Measurement of mycelia growth, conidiation, conidial germination, and appressorium formation. Mycelial growth was measured in six-well plates containing V8 agar (SPL Lifesciences Inc., Gyeonggi-Do, Korea) five days after inoculation with a mycelia agar plug (4 $\mathrm{mm}$ in diameter). The degree of conidiation was measured from ten-day-old oatmeal agar cultures in six-well plates. Conidia were collected with $5 \mathrm{ml}$ of distilled water and counted using a hemacytometer under a light microscope. Counting was done in three independent experiments with triplicates. Conidial germination and appressorium formation were measured on the hydrophobic side of a coverslip or GelBond film (FMC BioProducts, Rockland, ME, USA) as described previously (Lee and Dean, 1993). Conidia were harvested from six-day-old oatmeal agar cultures with sterile distilled water and adjusted to a concentration of $10^{4}$ conidia/ml. Drops (40 $\mu$ l of the conidial suspension) were placed on the hydrophobic side of the coverslips and incubated in a box containing pre-moistened wipes at $25^{\circ} \mathrm{C}$ for $8 \mathrm{~h}$. The percentages of conidial germination and appressorium formation from germinated conidia were determined by counting more than 100 conidia in at least four independent experiments with triplicate samples under a microscopy.

Pathogenicity assays. Conidia were harvested from ten- to twelve-day-old cultures on oatmeal agar plates with sterilized distilled water. A conidial suspension $\left(1 \times 10^{6}\right.$ conidia/ $\mathrm{ml}$ ) containing $250 \mathrm{ppm}$ Tween 20 was used for the spray inoculation of seedlings of a susceptible rice cultivar (Oryza sativa cv. Nakdong). The inoculated plants were placed in a moisture chamber at $25^{\circ} \mathrm{C}$ for $24 \mathrm{~h}$ in the dark, and then transferred to a growth chamber with one photoperiod of 16/8-h (light/dark). Disease severity was assessed seven days after inoculation based on the diseased leaf area (DLA) and lesion types, as described previously (Koh, 1986). For the infiltration assay, $100 \mu$ of a conidial suspension $\left(5 \times 10^{4}\right.$ conidia $\left./ \mathrm{ml}\right)$ was injected using a syringe without a needle onto the leaves of three-week-old rice plants. Disease symptoms were observed at seven days after inoculation. For the sheath infection assay, a spore suspension $\left(10^{5} / \mathrm{ml}\right.$ in $0.25 \%$ gelatin) was placed under the leaf sheaths of five-week-old rice plants. The incubated plants were covered with a plastic bag to maintain high humidity and laid down horizontally inside. At $48 \mathrm{~h}$ after inoculation, the sheaths were trimmed to produce epidermal layers above the midvein and used for microscopic obser- 
vation (Chi et al., 2009b; Koga et al., 2004). The assays for pathogenicity were repeated three times using triplicate samples.

Nucleic acid manipulation and polymerase chain reaction (PCR). Genomic DNA was isolated as described previously (Kim et al., 2005). Restriction enzyme digestion, agarose gel fractionation, cloning, and DNA gel blotting were performed using standard methods (Sambrook et al., 1989). Briefly, fungal genomic DNA was digested with SacI, separated on a $0.7 \%$ agarose gel, and transferred onto a Hybond $\mathrm{N}+$ membrane (Amersham Pharmacia Biotech, Little Chalfont, UK). The probes used for DNA hybridization were labeled with $\left[\alpha-{ }^{32} \mathrm{P}\right]-\mathrm{dCTP}$ using the Rediprime $^{\mathrm{TM}}$ II Random Prime Labeling System (Amersham Pharmacia Biotech, Piscataway, NJ, USA) according to the manufacturer's instructions. The hybridization membrane was exposed to a phosphorimager (BAS-2040, Fuji Photo Film, Tokyo, Japan) and visualized using a phosphorimager software. Total RNA was isolated from the mycelia using an Easy-Spin ${ }^{\mathrm{TM}}$ RNA extraction kit (iNtRON Biotechnology, Seoul, Korea). For reverse transcription-PCR (RTPCR), $3 \mu \mathrm{g}$ of total RNA were reverse-transcribed into first-strand cDNA using oligo (dT) primers and the SuperScript $^{\mathrm{TM}}$ First-Strand Synthesis System (Invitrogen Life Technologies, Carlsbad, CA, USA) according to the manufacturer's instructions. RT-PCR was performed on a PerkinElmer 9600 DNA thermal cycler in a $20-\mu 1$ reaction mixture containing $1 \mu \mathrm{l}$ of cDNA, $1 \mu \mathrm{l}$ of deoxyribonucleoside triphosphates ( $2.5 \mathrm{mM}$ dNTP mix), $2 \mu \mathrm{l}$ of $10 \times$ PCR buffer, $100 \mathrm{nM}$ each primer, and $1 \mathrm{U}$ of Taq polymerase. Real-time quantitative RT-PCR (qRT-PCR) was performed as described previously (Kim et al., 2005) using the AB7500 RealTime PCR system (Applied Biosystems, Foster City, CA, USA). The conditions included $3 \mathrm{~min}$ at $95^{\circ} \mathrm{C}$ ( 1 cycle $)$ followed by $15 \mathrm{~s}$ at $95^{\circ} \mathrm{C}, 30 \mathrm{~s}$ at $60^{\circ} \mathrm{C}$, and $30 \mathrm{~s}$ at $72^{\circ} \mathrm{C}(40$ cycles). Each qRT-PCR mixture (final volume $10 \mu \mathrm{l}$ ) contained $5 \mu$ of Power SYBR ${ }^{\circledR}$ Green PCR Master Mix (Applied Biosystems), $3 \mu$ of forward and reverse primers (100 $\mathrm{nM}$ each), and $2 \mu \mathrm{l}$ of cDNA (12.5 ng/ $\mu \mathrm{l})$. The oligonucleotide sequences (SDR1-RT for RT-PCT and SDR1-Q for qRT-PCR) are listed in Table 1. To compare the relative abundance of target gene transcripts, the average threshold cycle $(\mathrm{Ct})$ was normalized to that of $\beta$ tubulin for the samples as follows: $2^{-\Delta \mathrm{Ct}}$, where $-\Delta \mathrm{Ct}=$ $\left(C_{t, \text { target gene }}-C_{t, \beta \text {-tubulin }}\right)$. The fold-change for a target gene during fungal development, as compared to its growth in liquid complete medium, was calculated as $2^{-\Delta \Lambda \mathrm{Ct}}$, where $-\Delta \Delta C t=\left(C_{t, \text { target gene }}-C_{t, \beta \text {-tubulin }}\right)_{\text {test condition }}-\left(C_{t, w T}-C_{t, \beta \text {-tubulin }}\right)_{C M}$ (Livak and Schmittgen, 2001). qRT-PCR was performed twice using three independent tissue pools. The flanking region of the T-DNA was obtained by thermal asymmetric
interlaced-PCR (TAIL-PCR) as described previously (Liu and Whittier, 1995).

Targeted disruption of MoSDR1. The targeted gene disruption vector for $M O S D R 1$ was constructed by doublejoint PCR as described previously (Gritz and Davis, 1983; Yu et al., 2004). Fragments of the 5'-(1,263 bp) and 3'flanking regions $(1,264 \mathrm{bp})$ of the gene were amplified using the primers UF/UR and DF/DR, respectively (Table 1). A 2.1-kb hygromycin phosphotransferase gene $(H P H)$ cassette was amplified using primers HPHF and HPHR (Table 1) from pBCATPH (Gritz and Davies, 1983). The replacement construct was amplified with primers UF/DR using the fused product as the template. Protoplasts from strain KJ201 were transformed directly with the purified deletion construct after amplification. Hygromycin-resistant transformants were selected on TB3 $(0.3 \%$ yeast extract, $0.3 \%$ casamino acid, $1 \%$ glucose, and $20 \%$ sucrose) medium supplemented with $200 \mu \mathrm{g} / \mathrm{ml}$ hygromycin B (Calbiochem, San Diego, CA, USA) and screened by PCR. Putative two knock-out mutants were confirmed by DNA blotting and RT-PCR. Since the mutants were phenotypically indistinguishable, a representative mutant named as $\Delta M o s d r 1$ was used in this study.

FUN-1 staining. Conidial viability was examined by staining with the fluorescent dye FUN-1 (Molecular Probes, Eugene, OR, USA). A conidial suspension $\left(1 \times 10^{8}\right.$ conidia/ $\mathrm{ml}$ in $1 \%$ glucose, $10 \mathrm{mM} \mathrm{Na-HEPES}$, and $20 \mu \mathrm{M}$ FUN-1, $\mathrm{pH} 7.2$ ) was incubated at $30^{\circ} \mathrm{C}$ in the dark for $60 \mathrm{~min}$, then observed under optics equipped with epifluorescence (Millard et al., 1997). Stained conidia were viewed and photographed with excitation at 450-490 nm and emission at 520 nm under an Axioplan Universal microscope (Carl Zeiss Microscope Division, Oberkochen, Germany) equipped with a fluorescein filter set.

\section{Results}

MoSDR1 ${ }^{\text {T-DNA }}$ is a pathogenicity-defective T-DNA mutant of M. oryzae. We previously established a T-DNA insertional library via ATMT, which generated 21,070 hygromycinresistant mutants (Jeon et al., 2007). ATMT0879A1, a pathogenicity-defective mutant, was identified using a high-throughput screening system. As shown in Fig. 1A, ATMT0879A1, which is referred as MoSDR1 ${ }^{\text {T-DNA }}$, was nearly nonpathogenic; it produced a few tiny lesions that failed to develop further, whereas the wild-type strain caused typical symptoms of rice blast on the susceptible rice cultivar Nakdong. Assessment of pathogenicity according to the method of Koh et al. (1987) revealed that MoSDR1 $1^{\text {T-DNA }}$ significantly reduced the disease severity compared to wild- 

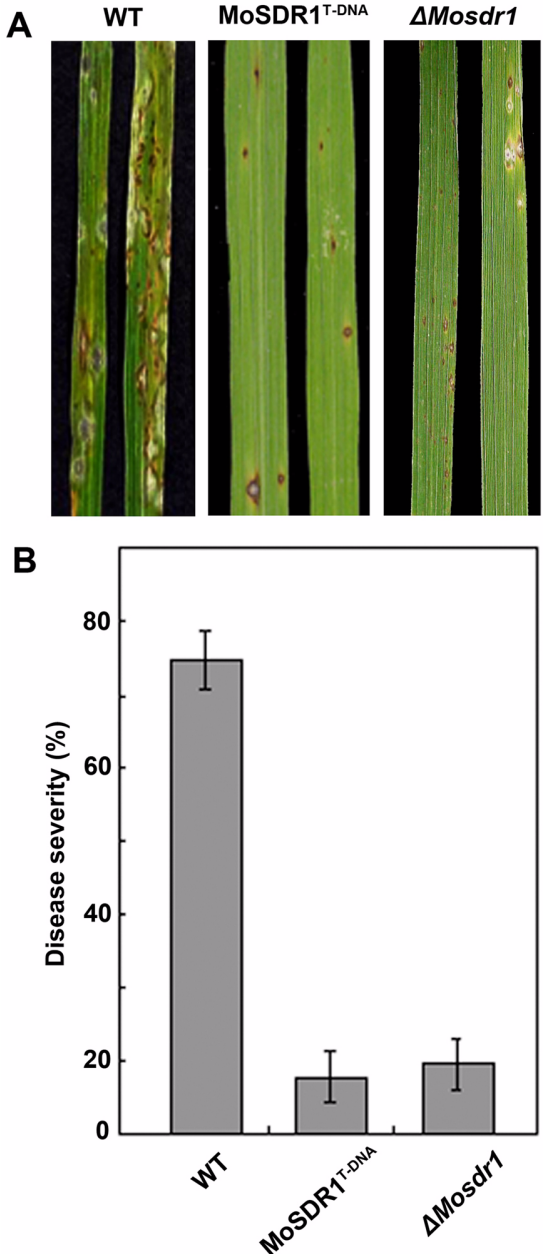

Fig. 1. Pathogenicity test of the $M$. oryzae mutants MoSDR $1^{\text {T.DNA }}$ and $\Delta M o s d r 1$. (A) Disease development in rice. Seedlings of a susceptible rice cultivar (cv. Nakdong) were inoculated with conidia $\left(1 \times 10^{6} / \mathrm{ml}\right)$ of the indicated strains. Photos of the inoculated leaves were taken seven days after spray inoculation. (B) Disease severity was measured based on the diseased leaf area and lesion types. The values given are the means \pm SD from three replicates inoculated by each strain. Three rice plants were used for each replicate.

type (Fig. 1B).

Southern blot analysis revealed that integration of a single copy of the T-DNA occurred in MoSDR $1^{\text {T-DNA }}$ (data not shown). The right flanking sequence of the T-DNA was determined by TAIL-PCR as described previously (Choi et al., 2007; Liu and Whittier, 1995). The results indicated that the T-DNA was inserted into the promoter region of an annotated gene, MGG00056 in supercontig 6.21 of the genome sequence. These data allowed us to create a schematic diagram of the integration of the T-DNA in ATMT0879A1 (Fig. 2A). RT-PCR revealed that the expression level of MGG00056 was reduced by two-fold in MoSDR1 $^{\text {T-DNA }}$, whereas the expression level of MGG11682 upstream of MGG00056 was unaffected compared to that
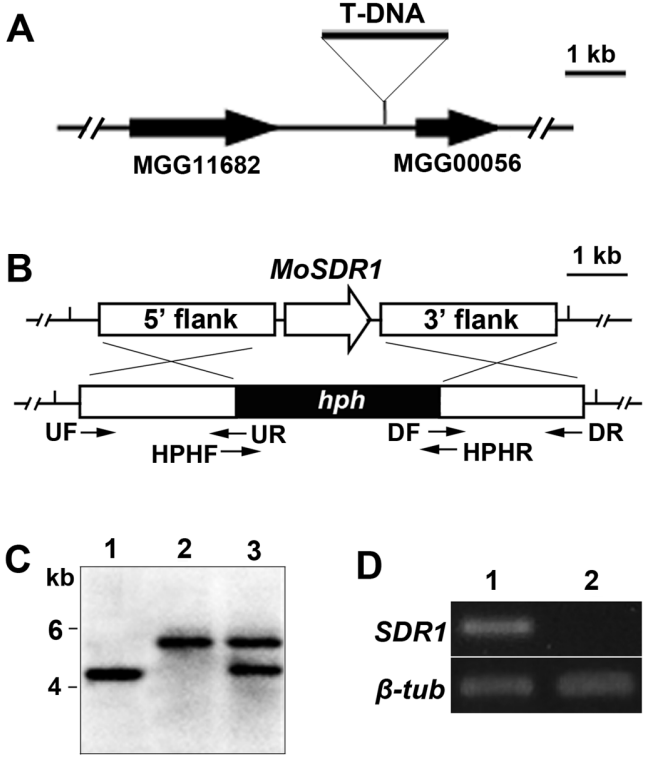

Fig. 2. Schematic diagram of the T-DNA insertion in ATMT0879A1 and the targeted deletion of MoSDR1. (A) Schematic diagram of the T-DNA insertion in ATMT0879A1. The insertion is located $271 \mathrm{bp}$ from the start codon in MGG00056. (B) Schematic diagram showing the targeted deletion of MoSDR1. The MoSDR1 replacement vector contained the homologous sequences flanking $h p h$ for homology-dependent replacement. S and closed arrows indicate the $\mathrm{SacI}$ restriction site and primer positions, respectively. (C) Genomic DNA was isolated from wild-type (lane 1), $\Delta M o s d r 1$ (lane 2), and an ectopic transformant (lane 3), digested with SacI, and probed with the 3'-flanking region. (D) MoSDR1 expression in the wild-type and $\Delta M o s d r l$. Total RNA was isolated from the mycelia of wild-type (lane 1) and $\Delta M o s d r 1$ cells (lane 2), then subjected to RT-PCR.

in the wild-type strain (data not shown). These data suggest that the pathogenicity defect of MoSDR $1^{\text {T-DNA }}$ results from functional interference with the annotated gene MGG00056.

MGG00056 encodes a member of the SDR family. A comparison of the cDNA sequence of MGG00056 with the genomic sequence confirmed that the gene consists of two exons and one intron and encodes a protein consisting of 286 amino acids with a predicted molecular mass of 29 kDa. MGG00056 was found to encode an SDR from a series of database searches, including BLASTP and PSORT (http://db.psort.org); it is hereafter referred to as MoSDRl. MoSDR1 showed significant similarity to predicted proteins from filamentous fungi, including 73 and $72 \%$ identity with proteins from Aspergillus oryzae and Penicillium chrysogenum, respectively (Fig. 3). MoSDR1 possesses an atypical NAD/NADH-binding motif (TGxxxxGxG instead of TGxxxGxG) and a typical catalytic site (YxxS/T-A/K), similar to SDR family members found in prokaryotic and eukaryotic organisms. The variability detected in the binding motif of MoSDR1 has been reported in other homologs 

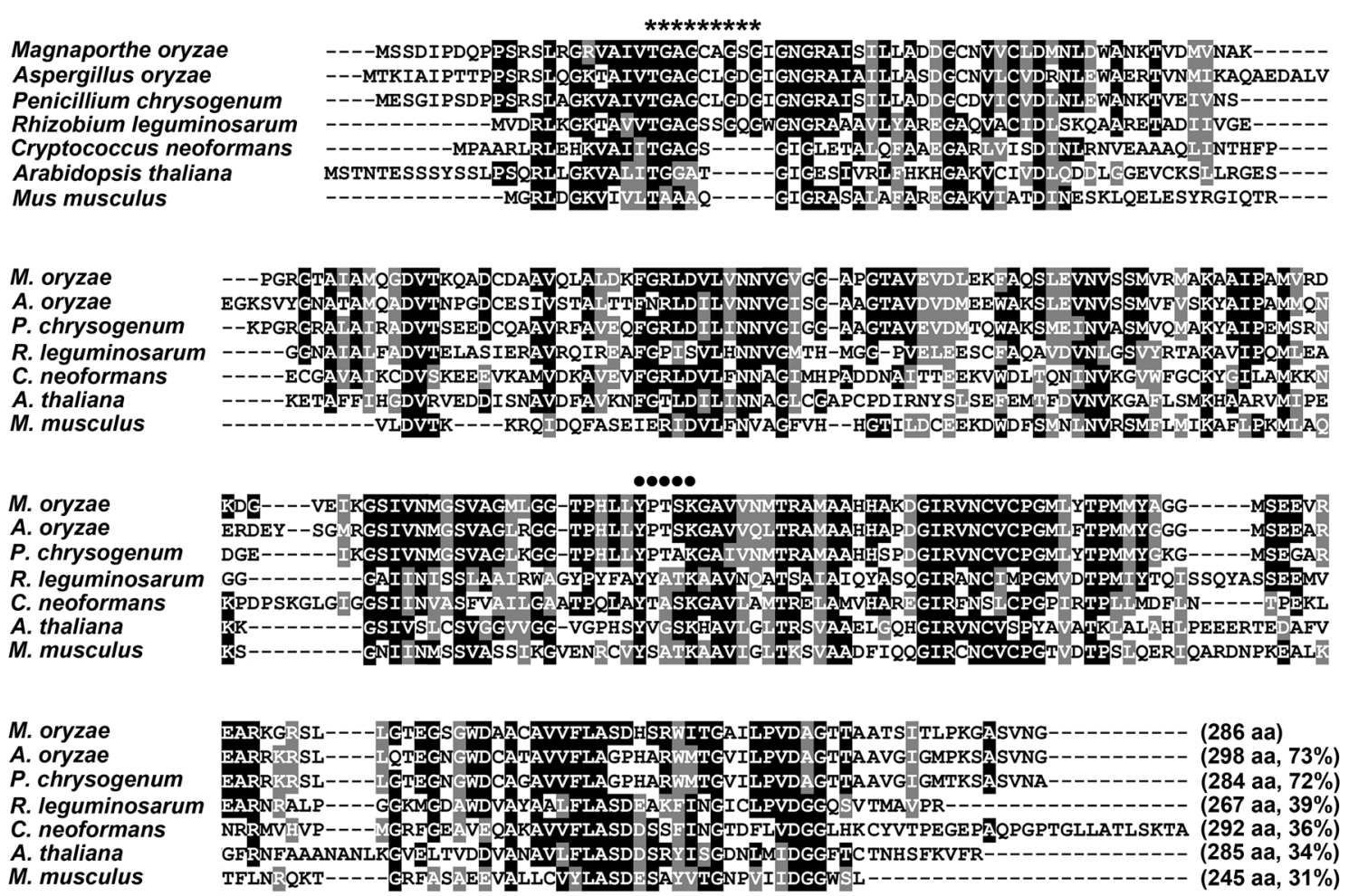

Fig. 3. Alignment of MoSDR1 with related proteins from Aspergillus oryzae (XP001821548), Penicillium chrysogenum (CAP96856), Rhizobium leguminosarum (YP002283857), Cryptococcus neoformans (XP568778), Arabidopsis thaliana (NP175644), and Mus musculus (NP081484). Blocks of identical or similar amino acids are marked by black or gray backgrounds, respectively. Asterisks and closed circles represent the coenzyme-binding motif and catalytic site, respectively.

(Jörnvall et al., 1995). The SDR family includes classical and extended types that differ in terms of their lengths and cofactor-binding motifs (Kallberg et al., 2002).

Targeted deletion of MoSDR1 reveals its role in disease development. To determine the function of MoSDR1, the coding region of $M O S D R 1$ was replaced with a hygromycin $\mathrm{B}$ expression cassette via a homology-dependent replacement strategy (Fig. 2B). Purified MoSDRl deletion constructs after amplification were used to transform fungal protoplasts. Hygromycin-resistant transformants were selected on a regeneration medium containing $200 \mu \mathrm{g} / \mathrm{ml}$ hygromycin B and screened by a PCR using the primers DR/ HPHF (Table 1). An MoSDR1 deletion mutant, $\triangle M o s d r 1$ was confirmed by Southern blot analysis and RT-PCR (Fig. $2 \mathrm{C}$ and $\mathrm{D}$ ). We first compared the pathogenicity of $\Delta M o s d r 1$ with that of MoSDR1 $1^{\text {T-DNA }}$. As expected, $\Delta M o s d r 1$ showed remarkably reduced pathogenicity compared to wild-type, similar to MoSDR ${ }^{\text {T-DNA }}$ (Fig. 1). This result indicates that MoSDR1 is required for M. oryzae pathogenicity.

MoSDR1 is required for conidial development. Quantitative RT-PCR revealed that MoSDRI mRNAs were highly expressed in conidia ( $>3$-fold), appressoria $(>2$ -
Table 1. List of primers used in this study

\begin{tabular}{ll}
\hline \hline Name & Sequence (5' to 3') \\
\hline MoSDR1 & \\
UF & GCATCCCAGTCGACATTTT \\
& GCACAGGTACACTTGTTTAGAGAGAT- \\
& GAGATCCAGTGTGGGCT \\
DF & CCTTCAATATCATCTTCTGTCGATGCCA- \\
DR & GACCTTTGTTCTCCT \\
SDR1-RTF & CTCCCTAAACTATCCTAGCCGACGAC \\
SDR1-RTR & AATGATCCGAAGCAAGAAAC \\
SDR1-QF & AGGTCAGAGAGGCCCGCAA \\
SDR1-QR & CCATTGACGCTCGCTCCTTTG \\
Hygromycin phosphotransferase \\
HPHF & CGACAGAAGATGATATTGAAGG \\
HPHR & CTCTAAACAAGTGTACCTGTGC \\
$\beta$-tubulin & \\
$\beta$-tuRTF & CTCCAGGGTTTCCAGATCAC \\
$\beta$-tuRTR & CCTCACCAGTGTACCAATGC \\
$\beta$-tuQF & TCGACAGCAATGGAGTTTACAAC \\
$\beta$-tuQR & AGCACCAGACTGACCGAAGAC \\
\hline
\end{tabular}

fold), and in planta ( $>3$-fold), relative to those in mycelia. To characterize the roles of MoSDR1 during M. oryzae 
Table 2. Comparison of phenotypic characteristics among the strains

\begin{tabular}{lcccc}
\hline \hline Strain & $\begin{array}{c}\text { Mycelial growth } \\
(\mathrm{mm})^{\mathrm{a}}\end{array}$ & $\begin{array}{c}\text { Conidiation } \\
\left(10^{5} / \mathrm{ml}\right)^{\mathrm{b}}\end{array}$ & $\begin{array}{c}\text { Conidial germination } \\
(\%)^{\mathrm{c}}\end{array}$ & $\begin{array}{c}\text { Appressorium formation } \\
(\%)^{\mathrm{d}}\end{array}$ \\
\hline Wild-type & $3.1 \pm 0.1$ & $17.4 \pm 1.1$ & $98.0 \pm 1.0$ & $96.4 \pm 1.5$ \\
MoSDR1 $1^{\text {T-DNA }}$ & $2.7 \pm 0.2$ & $0.6 \pm 0.1$ & $15.4 \pm 0.5$ & $3.2 \pm 0.8$ \\
$\Delta$ Mosdr1 & $2.6 \pm 0.1$ & $0.7 \pm 0.2$ & $17.4 \pm 0.8$ & $4.3 \pm 1.5$ \\
\hline
\end{tabular}

${ }^{a}$ Mycelial growth was measured at five days post-inoculation on V8 agar medium. The data are presented as the means \pm SD of three independent experiments with triplicates.

${ }^{\mathrm{b}}$ Conidiation was measured by counting the number of conidia collected from ten-day-old oatmeal agar cultures in six-well plates.

${ }^{\mathrm{c}}$ The percentage of conidial germination on a hydrophobic surface was measured under a light microscope using conidia harvested from six-dayold oatmeal agar plates.

${ }^{\mathrm{d}}$ The percentage of appressorium formation from germinated conidia on a hydrophobic surface was measured using conidia harvested from sixday-old oatmeal agar plates.

development, phenotypic characterization of the strains was carried out. Measurements of mycelial growth showed that $\Delta M o s d r l$ was slightly retarded compared to wild-type on complete medium, similar to MoSDR1 $1^{\text {T-DNA }}$ (Table 2). The morphologies of the colonies of the two strains were indistinguishable (data not shown). Quantitative measurement of the conidia indicated that conidial production was significantly reduced in MoSDR $1^{\text {T-DNA }}$. As expected, the deletion of MoSDR1 resulted in a significant reduction in conidiation as shown in MoSDR ${ }^{1-D N A}$. These results indicate that MoSDR1 plays a role in asexual (conidial) reproduction.

\section{MoSDR1 is indispensible for preinfection-related develop-} ment and pathogenicity. The germination of MoSDR $1^{\text {TDNA }}$ conidia was significantly reduced, whereas about $98 \%$ of wild-type conidia germinated (Table 2). Therefore, we tested whether the deletion of MoSDR1 affects this stage of preinfection development. A large proportion $(>80 \%)$ of $\ddot{A} M o s d r 1$ conidia failed to germinate on a hydrophobic surface (Table 2). After conidial germination, the wild-type strain formed dome-shaped appressoria at the ends of the germ tubes after $6 \mathrm{~h}$ of incubation on a hydrophobic surface. More than $95 \%$ of the wild-type conidia formed appressoria (Table 2); however, appressorium development in both $\triangle M o s d r 1$ and MoSDR $1^{\text {T-DNA }}$ was significantly reduced.

To determine whether the $\Delta M o s d r 1$ mutant exhibited invasive growth in plant cells, a conidial suspension was infiltrated into rice leaves by injection using a syringe. The wild-type strain produced blast lesions, whereas $\Delta M o s d r 1$ failed to cause the disease. These data indicate that the mutant lost the ability to grow inside plant cells (Fig. 4A). To test the role of MoSDR1 in penetration, rice leaf sheath cells were inoculated with a conidial suspension. The wildtype strain penetrated the epidermal cells of the leaves and grew invasively (Fig. 4B). In contrast, the $\Delta M o s d r 1$ mutant was unable to penetrate the plant surface, showing hyphal growth on all surfaces. These results suggest that the functioning of MoSDR 1 is required for appressorium-mediated penetration and further growth inside the host.
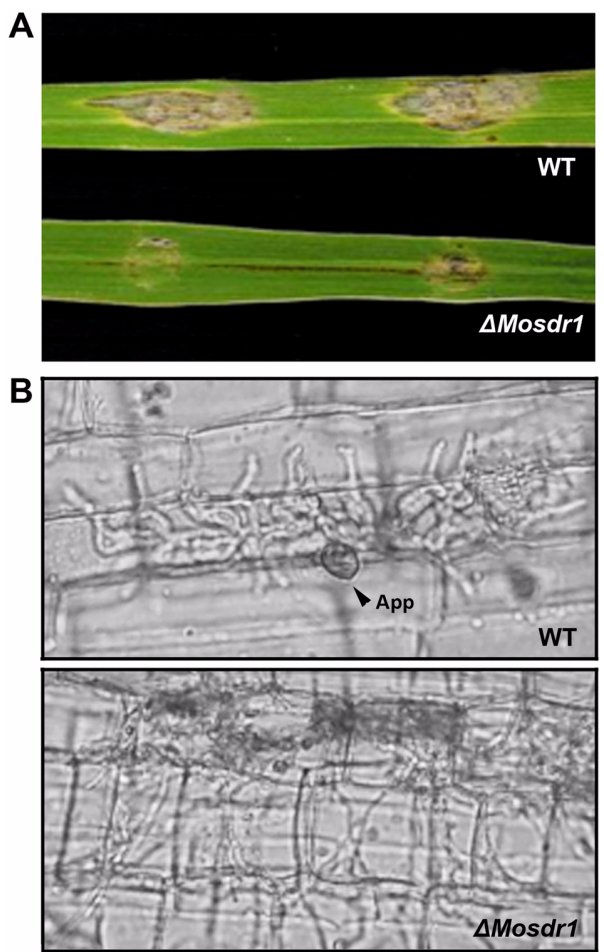

Fig. 4. In planta assays for fungal growth and penetration. (A) Infiltration assay for invasive growth inside plant cells. Conidial suspensions $\left(5 \times 10^{4}\right.$ conidia/ml $)$ were infiltrated into rice leaves. Photographs were taken seven days after inoculation. (B) Sheath inoculation assay. Forty microliters of a conidial suspension $\left(3 \times 10^{4} / \mathrm{ml}\right)$ of the strains were inoculated onto rice sheath cells for $48 \mathrm{~h}$. The arrowhead indicates appressorium (App).

MoSDR1 is required for conidial viability. To determine whether the inability of the $\Delta M o s d r 1$ conidia to germinate was associated with conidial viability, we stained the conidia with the dye FUN-1. Briefly, conidia were freshly harvested in a solution ( $2 \%$ glucose and $10 \mathrm{mM}$ HEPES, $\mathrm{pH}$ 7.2) from seven-day-old oatmeal agar plates, and incubated at $30^{\circ} \mathrm{C}$ for $60 \mathrm{~min}$ in dark. Most of the wild-type conidia ( $>97 \%)$ exhibited orange fluorescence, indicating that they were metabolically active; in contrast, most of the 


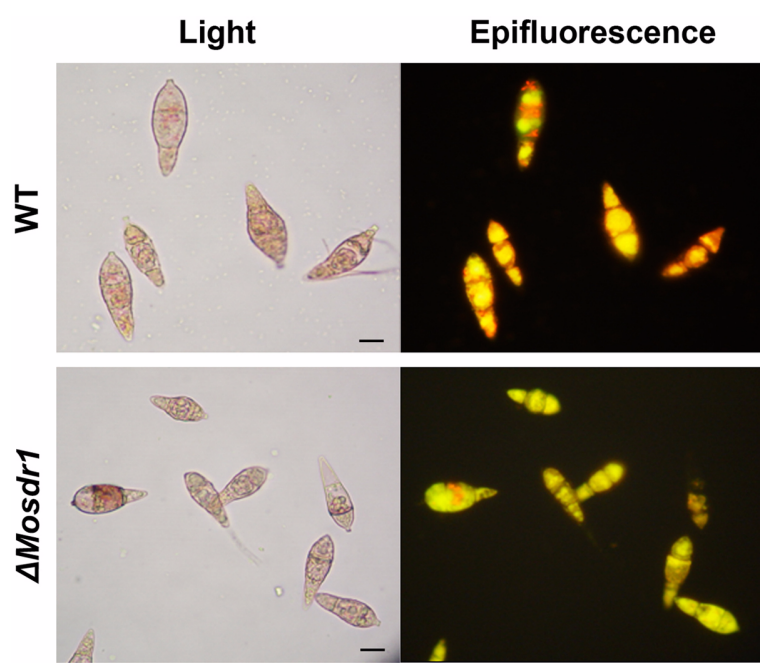

Fig. 5. Assay for conidial viability. Conidia were stained with the fluorescent dye FUN-1, and observed under a light or epifluorescence microscope. Bars $=10 \mu \mathrm{m}$.

$\Delta M o s d r 1$ conidia (>82\%) exhibited green fluorescence, indicating that most of the $\Delta M o s d r 1$ conidia were dead cells (Fig. 5). This observation is positively correlated with the conidial germination rate of the wild-type and $\Delta M o s d r 1$ strains.

\section{Discussion}

During the course of a mutant screen to elucidate the molecular mechanisms underlying pathogenicity, we isolated candidates unable to cause rice blast in susceptible rice plants (Jeon et al., 2007). In particular, the MoSDR1 $1^{\text {T-DNA }}$ mutant showed a severe loss of virulence. Molecular cloning of MoSDR $1^{\text {T-DNA }}$ revealed that the corresponding gene encodes a new member of the SDR family. The targeted deletion of MoSDR1 resulted in a significant reduction in pathogenicity, as seen in MoSDR $1^{\text {T-DNA }}$. The loss of pathogenicity was not solely due to the lack of appressoria because wounded leaflets inoculated with mutants did not develop rice blast inside host cells. Together with the consistency of the phenotypes of MoSDR $1^{\text {TDNA }}$ and $\Delta M o s d r 1$, these results indicate that the multimeric nature of the SDRs in M. oryzae does not functionally complement MoSDR1 in the deletion mutant.

SDRs encompass a large group of functionally diverse proteins in organisms (Jörnvall et al., 1995). Members in the SDR family generally exhibit low pair-wise sequence identity of only about $12-30 \%$, probably because of their early divergence and remote origin (Jörnvall et al., 1995). This may alternatively suggest that SDRs have substrate specificity. For instance, the two SDR enzymes 3HNR and 4HNR that catalyze the same naphthol reduction reactions in melanin biosynthesis pathway in M. oryzae exhibit considerably different substrate specificities (Thompson et al., 1997; Thompson et al., 2000). The melanin biosynthesis plays a crucial role in $M$. oryzae pathogenic development; the infection structure, appressorium must be melanized. Consequently, 3HNR is the biochemical target of commercial agricultural fungicides (tricyclazole, pyroquilon, and phthalide) to prevent rice blast (Tokousbalides and Sisler, 1978; Woloshuk et al., 1980; Woloshuk and Sisler, 1982). Unlike melanin-deficient mutants of $M$. oryzae lacking 3HNR and 4HNR expression (Chumley and Valent, 1990), the melanization in MoSDR1 deletion mutant appeared to be normal (data not shown).

MoSDR1 deletion mutant was significantly defective in conidial reproduction whereas its mycelial growth was not affected, indicating the requirement of MoSDR1 in conidiation. This result correlates with the observation that the expression of MoSDR1 was dramatically induced during conidiation. This implies that the event of conidiation is mediated via the transcriptional regulation of MoSDR1 gene, rather than direct regulation of MoSDR1 activity. Consistent with this idea was the observation that phenotypic defects in the MoSDR1 $1^{\text {T-DNA }}$ were quite similar to those in the $\Delta M o s d r 1$. Development of conidia, as propagules, is a key step to ensure fungal survival and infectability. In general, fungi terminate conidial development through desiccation after all the requirements are prepared in conidia. Although conidial morphology of MoSDR1 deletion mutant was indistinguishable from the wild-type conidia, MoSDR1 conidia failed to germinate. The germination defect of $\Delta M o s d r 1$ conidia may not be caused by their inability to sense environmental factors such as nutrients and moisture, but by a metabolic abnormality in the process of conidial development. In a histochemical study, we showed this to be the case that the $\Delta M o s d r 1$ conidia lost viability. In addition, appressorium development was completely abolished on germinated conidia of the $\Delta M o s d r 1$. These results suggest that MoSDR1-mediated metabolic regulation is indispensible not only for pre-infection stages, but for pathogenic development.

MoSDR1 showed a high similarity to filamentous fungal proteins. It is tempting to speculate that the MoSDR1 and its homologous proteins have a common enzymatic mechanism among filamentous fungi. SDRs utilize a wide range of substrates, including steroids, alcohols, sugars, and aromatic compounds. The substrate of MoSDR1, which may be rich in conidia based on the phenotypes of $\triangle M o s d r 1$ and the expression patterns of MoSDR1, remains to be characterized. The next challenge will be to uncover how MoSDR1 biogenesis is involved in M. oryzae conidiation and conidial viability. Understanding these processes will lead to development of a novel strategy for the control of phytopathogenic fungal diseases. 


\section{Acknowledgments}

This work was supported by the National Research Foundation of Korea grants (2009-0063340 and 20090080161) and grants from the Biogreen21 (20080401-034044-009-01-00), the TDPAF (309015-04-SB020), and the Crop Functional Genomics Center (2009K001198). KSK is grateful for the fellowship through the Brain Korea 21 Program.

\section{References}

Butchko, R. A. E., Plattner, R. D. and Proctor, R. H. 2003. FUM13 encodes a short chain dehydrogenase/reductase required for C-3 carbonyl reduction during fumonisin biosynthesis in Gibberella moniliformis. J. Agric. Food Chem. 51:3000-3006.

Cheng, W. H., Endo, A., Zhou, L., Penney, J., Chen, H. C., Arroyo, A., Leon, P., Nambara, E., Asami, T., Seo, M., Koshiba, T. and Sheen, J. 2002. A unique short-chain dehydrogenase/reductase in Arabidopsis glucose signaling and abscisic acid biosynthesis and functions. Plant Cell 14:2723-2743.

Chi, M. H., Park, S. Y., Kim, S. and Lee, Y. H. 2009a. A quick and safe method for fungal DNA extraction. Plant Pathol. J. 25:108-111.

Chi, M. H., Park, S. Y., Kim, S. and Lee, Y. H. 2009b. A novel pathogenicity gene is required in the rice blast fungus to suppress the basal defenses of the host. PLoS Pathog. 5:e1000401.

Choi, J., Kim, Y., Kim, S., Park, J. and Lee, Y. H. 2009. MoCRZ1, a gene encoding a calcineurin-responsive transcription factor, regulates fungal growth and pathogenicity of Magnaporthe oryzae. Fungal Genet. Biol. 46:243-254.

Choi, J., Park, J., Jeon, J., Chi, M. H., Goh, J., Yoo, S. Y., Jung, K., Kim, H., Park, S. Y., Rho, H. S., Kim, S., Kim, B. R., Han, S. S., Kang, S. and Lee, Y. H. 2007. Genome-wide analysis of TDNA integration into the chromosomes of Magnaporthe oryzae. Mol. Microbiol. 66:371-382.

Choi, J. H., Kim, Y. and Lee, Y. H. 2009. Functional analysis of $M C N A$, a gene encoding a catalytic subunit of calcineurin, in the rice blast fungus Magnaporthe oryzae. J. Microbiol. Biotechnol. 19:11-16.

Choi, W. and Dean, R. A. 1997. The adenylate cyclase gene MACl of Magnaporthe grisea controls appressorium formation and other aspects of growth and development. Plant Cell 9:1973-1983.

Chumley, F. G. and Valent, B. 1990. Genetic analysis of melanindeficient, nonpathogenic mutants of Magnaporthe grisea. Mol. Plant-Microbe Interact. 3:135-143.

Dean, R. A., Talbot, N. J., Ebbole, D. J., Farman, M. L., Mitchell, T. K., Orbach, M. J., Thon, M., Kulkarni, R., Xu, J. R., Pan, H. Q., Read, N. D., Lee, Y. H., Carbone, I., Brown, D., Oh, Y. Y., Donofrio, N., Jeong, J. S., Soanes, D. M., Djonovic, S., Kolomiets, E., Rehmeyer, C., Li, W. X., Harding, M., Kim, S., Lebrun, M. H., Bohnert, H., Coughlan, S., Butler, J., Calvo, S., Ma, L. J., Nicol, R., Purcell, S., Nusbaum, C., Galagan, J. E. and Birren, B. W. 2005. The genome sequence of the rice blast fungus Magnaporthe grisea. Nature 434:980-986.

DeLong, A., Calderon-Urrea, A. and Dellaporta, S. L. 1993. Sex determination gene TASSELSEED2 of maize encodes a shortchain alcohol dehydrogenase required for stage-specific floral organ abortion. Cell 74:757-768.

Gritz, L. and Davies, J. 1983. Plasmid-encoded hygromycin B resistance: the sequence of hygromycin B phosphotransferase gene and its expression in Escherichia coli and Saccharomyces cerevisiae. Gene 25:179-188.

Howard, R. J., Ferrari, M. A., Roach, D. H. and Money, N. P. 1991. Penetration of hard substrates by a fungus employing enormous turgor pressures. Proc. Natl. Acad. Sci. US A 88:11281-11284.

Jörnvall, H., Hoog, J. O. and Persson, B. 1999. SDR and MDR: completed genome sequences show these protein families to be large, of old origin, and of complex nature. FEBS Lett. 445:261-264.

Jörnvall, H., Persson, B., Krook, M., Atrian, S., Gonzalez-Duarte, R., Jeffery, J. and Ghosh, D. 1995. Short-chain dehydrogenases/reductases (SDR). Biochemistry 34:6003-6013.

Jeon, J., Goh, J., Yoo, S., Chi, M. H., Choi, J., Rho, H. S., Park, J., Han, S. S., Kim, B. R., Park, S. Y., Kim, S. and Lee, Y. H. 2008. A putative MAP kinase kinase kinase, $M C K 1$, is required for cell wall integrity and pathogenicity of the rice blast fungus, Magnaporthe oryzae. Mol. Plant-Microbe Interact. 21: 525-534.

Jeon, J., Park, S. Y., Chi, M. H., Choi, J., Park, J., Rho, H. S., Kim, S., Goh, J., Yoo, S., Park, J. Y., Yi, M., Yang, S., Kwon, M. J., Han, S. S., Kim, B. R., Khang, C. H., Park, B., Lim, S. E., Jung, K., Kong, S., Karunakaran, M., Oh, H. S., Kim, H., Kang, S., Choi, W. B. and Lee, Y. H. 2007. Genome-wide functional analysis of pathogenicity genes in the rice blast fungus. Nat. Genet. 39:561-565.

Kallberg, Y., Oppermann, U., Jornvall, H. and Persson, B. 2002. Short-chain dehydrogenases/reductases (SDRs). Eur. J. Biochem. 269:4409-4417.

Kallberg, Y. and Persson, B. 2006. Prediction of coenzyme specificity in dehydrogenases/reductases: a hidden Markov modelbased method and its application on complete genomes. FEBS J. 273:1177-1184.

Kim, S., Ahn, I. P., Rho, H. S. and Lee, Y. H. 2005. MHP1, a Magnaporthe grisea hydrophobin gene, is required for fungal development and plant colonization. Mol. Microbiol. 57:12241237.

Koga, H., Dohi, K., Nakayachi, O. and Mori, M. 2004. A novel inoculation method of Magnaporthe grisea for cytological observation of the infection process using intact leaf sheaths of rice plants. Physiol. Mol. Plant Pathol. 64:67-72.

Koh, Y. J. 1986. Adult-plant resistance of rice cultivars to blast. Ph.D. thesis. Seoul National University, Suwon, Korea.

Lee, S. C. and Lee, Y. H. 1998. Calcium/calmodulin-dependent signaling for appressorium formation in the plant pathogenic fungus Magnaporthe grisea. Mol. Cells 8:698-704.

Lee, Y. H. and Dean, R. A. 1993. cAMP regulates infection structure formation in the plant-pathogenic fungus Magnaporthe grisea. Plant Cell 5:693-700. 
Liu, Y. G. and Whittier, R. F. 1995. Thermal asymmetric interlaced PCR: automatable amplification and sequencing of insert end fragments from P1 and YAC clones for chromosome walking. Genomics 25:674-681.

Livak, K. J. and Schmittgen, T. D. 2001. Analysis of relative gene expression data using real-time quantitative PCR and the $2^{-\Delta A \mathrm{a}}$ method. Methods 25:402-408.

Millard, P. J., Roth, B. L., Thi, H. P., Yue, S. T. and Haugland, R. P. 1997. Development of the FUN-1 family of fluorescent probes for vacuole labeling and viability testing of yeasts. Appl. Environ. Microbiol. 63:2897-2905.

Mitchell, T. K. and Dean, R. A. 1995. The cAMP-dependent protein kinase catalytic subunit is required for appressorium formation and pathogenesis by the rice blast pathogen Magnaporthe grisea. Plant Cell 7:1869-1878.

Oppermann, U. C., Filling, C. and Jörnvall, H. 2001. Forms and functions of human SDR enzymes. Chem. Biol. Interact. 130132:699-705.

Ou, S. H. 1985. Rice diseases. Surrey: Commonwealth Mycological Institute. pp. 97-184.

Persson, B., Kallberg, Y., Bray, J. E., Bruford, E., Dellaporta, S. L., Favia, A. D., Duarte, R. G., Jornvall, H., Kavanagh, K. L., Kedishvili, N., Kisiela, M., Maser, E., Mindnich, R., Orchard, S., Penning, T. M., Thornton, J. M., Adamski, J. and Oppermann, U. 2009. The SDR (short-chain dehydrogenase/reductase and related enzymes) nomenclature initiative. Chem. Biol. Interact. 178:94-98.

Rossmann, M. G. and Argos, P. 1978. The taxonomy of binding sites in proteins. Mol. Cell Biochem. 21:161-182.

Sambrook, J., Fritsch, E. F. and Maniatis, T. 1989. Molecular cloning: A laboratory manual. Cold Spring Harbor Laboratory Press, New York, USA.

Thompson, J. E., Basarab, G. S., Andersson, A., Lindqvist, Y. and Jordan, D. B. 1997. Trihydroxynaphthalene reductase from Magnaporthe grisea: realization of an active center inhibitor and elucidation of the kinetic mechanism. Biochemistry 36: 1852-1860.

Thompson, J. E., Fahnestock, S., Farrall, L., Liao, D. I., Valent, B. and Jordan, D. B. 2000. The second naphthol reductase of fungal melanin biosynthesis in Magnaporthe grisea: tetrahydroxynaphthalene reductase. J. Biol. Chem. 275:34867-34872.

Tokousbalides, M. C. and Sisler, H. D. 1978. Effect of tricyclazole on growth and secondary metabolism in Pyricularia oryzae. Pestic. Biochem. Physiol. 8:26-32.

Valent, B. 1990. Rice blast as a model system for plant pathology. Phytopathology 80:33-36.

Villarroya, A., Juan, E., Egestad, B. and Jörnvall, H. 1989. The primary structure of alcohol dehydrogenase from Drosophila lebanonensis. Extensive variation within insect 'short-chain' alcohol dehydrogenase lacking zinc. Eur. J. Biochem. 180:191197.

Woloshuk, C. P., Sisler, H. D., Tokousbalides, M. C. and Dutky, S. R. 1980. Melanin biosynthesis in Pyricularia oryzae: site of tricyclazole inhibition and pathogenicity of melanin-deficient mutants. Pest Biochem. Physiol. 14:256-264.

Woloshuk, C. P. and Sisler, H. D. 1982. Tricyclazole, pyroquilon, tetrachlorophthalide, PCBA, coumarin and related compounds inhibit melanization and epidermal penetration by Pyricularia oryzae. J. Pestic. Sci. 7:161-166.

Xu, J. R. and Hamer, J. E. 1996. MAP kinase and cAMP signaling regulate infection structure formation and pathogenic growth in the rice blast fungus Magnaporthe grisea. Genes Dev. 10:2696-2706.

Xu, J. R., Urban, M., Sweigard, J. A. and Hamer, J. E. 1997. The CPKA gene of Magnaporthe grisea is essential for appressorial penetration. Mol. Plant-Microbe Interact. 10:187-194.

Yu, J. H., Hamari, Z., Han, K. H., Seo, J. A., Reyes-Dominguez, Y. and Scazzocchio, C. 2004. Double-joint PCR: a PCR-based molecular tool for gene manipulations in filamentous fungi. Fungal Genet. Biol. 41:973-981. 